\title{
TENSOR AND INTEGRITY BASES FOR THE GYROIDAL CRYSTAL CLASS
}

\author{
BY \\ G. F. SMITH (Lehigh University)
}

1. Introduction. A tensor $c_{i j} \ldots k$ which satisfies the equations

$$
c_{i j} \ldots k=t_{i p} t_{i q} \cdots t_{k r} c_{p a} \cdots r
$$

for all transformations $\mathbf{T}=\left\|t_{i p}\right\|$ belonging to a group $\boldsymbol{\Gamma}$ is said to be invariant under $\boldsymbol{\Gamma}$. A set of tensors each of which is invariant under $\boldsymbol{\Gamma}$ and such that any tensor invariant under $\boldsymbol{\Gamma}$ is expressible as a linear combination of outer products of these tensors is said to form a tensor basis for $\mathbf{\Gamma}$. A function $W\left(\mathbf{x}_{1}, \mathbf{x}_{2}, \cdots, \mathbf{x}_{N}\right)$ of the vectors $\mathbf{x}_{1}, \mathbf{x}_{2}, \cdots, \mathbf{x}_{N}$ which satisfies the equations

$$
W\left(\mathbf{T} \mathbf{x}_{1}, \mathbf{T} \mathbf{x}_{2}, \cdots, \mathbf{T x}_{N}\right)=W\left(\mathbf{x}_{1}, \mathbf{x}_{2}, \cdots, \mathbf{x}_{N}\right)
$$

for all transformations $\mathbf{T}$ belonging to a group $\boldsymbol{\Gamma}$ is said to be invariant under $\boldsymbol{\Gamma}$. A set of polynomials $I_{p}\left(\mathbf{x}_{1}, \mathbf{x}_{2}, \cdots, \mathbf{x}_{N}\right)(p=1,2, \cdots, m)$, each of which is invariant under $\boldsymbol{\Gamma}$, is said to form an integrity basis for $N$ vectors under $\boldsymbol{\Gamma}$ if any polynomial function of the $N$ vectors which is invariant under $\boldsymbol{\Gamma}$ is expressible as a polynomial in $I_{1}, I_{2}, \cdots, I_{m}$. The $I_{p}$ are referred to as elements of the integrity basis.

The tensor basis and the integrity basis for $N$ vectors has been obtained by Smith and Rivlin [1] for each of the groups associated with the various crystal classes with the exception of the gyroidal class whose Schoenflies, Hermann-Maguin and Schubnikov symbols are 0,43 and $\frac{3}{4}$ respectively. In this note, we fill this gap in the literature by deriving the tensor basis and the integrity basis for $N$ vectors for the gyroidal class.

If an element $I_{m}$ (say) of the integrity basis is expressible as a polynomial in the remaining elements $I_{1}, \cdots, I_{m-1}$, then $I_{m}$ is said to be redundant. If none of the $I_{1}, \cdots, I_{m}$ are redundant, the integrity basis is said to be irreducible. We shall show in a later paper [2] that none of the multilinear elements of the integrity basis are redundant. Since the elements of the tensor basis are obtained directly from these multilinear invariants (see Sec. 3, Formula (3.1)), this will also imply the irreducibility of the tensor basis.

2. Integrity basis. The group $\boldsymbol{\Gamma}$ associated with the gyroidal class is comprised of the twenty-four transformations

$$
\left(I, D_{1}, D_{2}, D_{3}\right) \cdot\left(I, M_{1}, M_{2}\right), \quad\left(C, R_{1}, R_{2}, R_{3}\right) \cdot\left(T_{1}, T_{2}, T_{3}\right)
$$

where the transformations $I, D_{1}, \cdots, T_{3}$ are defined in [3]. The transformations (2.1) are proper orthogonal and consequently $\operatorname{det}\left(\mathbf{x}_{1}, \mathbf{x}_{2}, \mathbf{x}_{3}\right)$ is invariant under $\boldsymbol{\Gamma}$. It then follows from Peano's theorem (see [4, pp. 39-44] or [5, p. 261]) that an integrity basis for $N$ three-dimensional vectors may be derived from det $\left(\mathbf{x}_{1}, \mathbf{x}_{2}, \mathbf{x}_{3}\right)$ and the integrity basis for two vectors by the process of polarization. Thus, if $I_{1}, I_{2}, \cdots, I_{\mu}$ form an integrity basis for functions of two vectors $\mathbf{x}_{1}, \mathbf{x}_{2}$ invariant under $\boldsymbol{\Gamma}$, then an integrity basis for $N$ vectors is given by

Received August 12, 1966. 


$$
\operatorname{det}\left(\mathbf{x}_{K_{1}}, \mathbf{x}_{K_{2}}, \mathbf{x}_{K_{\mathbf{s}}}\right)
$$

and

$$
x_{i_{1}}^{\left(K_{1}\right)} x_{i_{2}}^{\left(K_{2}\right)} \cdots x_{i_{0}}^{\left(K_{0}\right)} \frac{\partial^{8} I_{\alpha}}{\partial x_{i_{1}}^{\left(L_{1}\right)} \partial x_{i_{2}}^{\left(L_{2}\right)} \cdots \partial x_{i_{0}}^{\left(L_{8}\right)}}
$$

where $\alpha=1, \cdots, \mu ; K_{1}, \cdots, K_{s}$ are integers chosen from $1, \cdots, N$ and $L_{1}, \cdots, L$, are appropriately chosen integers from 1,2 . The quantities $x_{i}^{(K)}$ represent the components of the vector $\mathbf{x}_{K}$.

We shall employ the notation $\sum$ to indicate the sum of the three quantities obtained by cyclic permutation of subscripts on the summand, thus:

$$
\sum x_{1} y_{1}=x_{1} y_{1}+x_{2} y_{2}+x_{3} y_{3} \text {. }
$$

It may be readily shown by the methods employed in [1] that the integrity basis for functions of two vectors $\mathbf{x}$ and $\mathbf{y}$ invariant under the gyroidal group is given by

$$
\sum x_{1}^{\alpha} y_{1}^{\beta}, \sum x_{1}^{\gamma} y_{1}^{\delta}\left(x_{2} y_{3}-x_{3} y_{2}\right)
$$

where $\alpha, \beta, \gamma, \delta$ are positive integers or zero such that $\alpha+\beta=2,4$ or $6 ; \gamma+\delta=3$ or 5 and

$$
y_{i_{1}} \cdots y_{i}, \partial^{\nu} \tau / \partial x_{i_{1}} \cdots \partial x_{i_{1}} \quad(\nu=0,1,2, \cdots, 9)
$$

where

$$
\tau=\sum x_{1}^{5}\left(x_{2}^{3} x_{3}-x_{3}^{3} x_{2}\right)
$$

The integrity basis for $N$ vectors is then obtained from (2.2) upon substituting the invariants (2.4) and (2.5) for $I_{1}, \cdots, I_{\mu}$ in (2.2). We list below the typical multilinear elements of this integrity basis.

Two vectors. $\quad \sum x_{1} y_{1}$.

Three vectors. $\quad \sum x_{1}\left(y_{2} z_{3}-y_{3} z_{2}\right)$.

Four vectors. $\quad \sum x_{:} y_{1} z_{1} u_{1}$.

Five vectors. $\quad \theta(x, y, z, u ; v), \theta(v, x, y, z ; u)$,

$$
\theta(u, v, x, y ; z), \theta(z, u, v, x ; y)
$$

where

$$
\begin{aligned}
\theta(x, y, z, u ; v)=\sum x_{1} y_{1} z_{1}\left(u_{2} v_{3}-u_{3} v_{2}\right) & +\sum x_{1} y_{1} u_{1}\left(z_{2} v_{3}-z_{3} v_{2}\right) \\
& +\sum x_{1} z_{1} u_{1}\left(y_{2} v_{3}-y_{3} v_{2}\right)+\sum y_{1} z_{1} u_{1}\left(x_{2} v_{3}-x_{3} v_{2}\right)
\end{aligned}
$$

Six vectors. $\quad \sum x_{1} y_{1} z_{1} u_{1} v_{1} w_{1}$.

Seven vectors. $\quad \phi(x, y, z, u, v, w ; r), \phi(r, x, y, z, u, v ; w)$,

$$
\begin{aligned}
& \phi(w, r, x, y, z, u ; v), \phi(v, w, r, x, y, z ; u), \\
& \phi(u, v, w, r, x, y ; z), \phi(z, u, v, w, r, x ; y)
\end{aligned}
$$


where

$$
\begin{aligned}
\phi(x, y, z, u, v, w ; r)=\sum x_{1} y_{1} z_{1} u_{1} v_{1}\left(w_{2} r_{3}-w_{3} r_{2}\right)+\sum x_{1} y_{1} z_{1} u_{1} w_{1}\left(v_{2} r_{3}-v_{3} r_{2}\right) \\
+\sum x_{1} y_{1} z_{1} v_{1} w_{1}\left(u_{2} r_{3}-u_{3} r_{2}\right)+\sum x_{1} y_{1} u_{1} v_{1} w_{1}\left(z_{2} r_{3}-z_{3} r_{2}\right) \\
+\sum x_{1} z_{1} u_{1} v_{1} w_{1}\left(y_{2} r_{3}-y_{3} r_{2}\right)+\sum y_{1} z_{1} u_{1} v_{1} w_{1}\left(x_{2} r_{3}-x_{3} r_{2}\right) .
\end{aligned}
$$

Nine vectors. $\quad x_{i_{1}} y_{i_{2}} z_{i_{3}} u_{i_{\mathrm{s}}} v_{i_{\mathrm{s}}} w_{i_{\mathrm{s}}} r_{i_{1}} s_{i_{\mathrm{s}}} t_{i_{\diamond}} \partial^{9} \tau / \partial x_{i_{1}} \partial x_{i_{2}} \cdots \partial x_{i_{\bullet}}$

where

$$
\tau=\sum x_{1}^{5}\left(x_{2}^{3} x_{3}-x_{3}^{3} x_{2}\right) .
$$

An integrity basis for $N$ vectors may be obtained by substituting the vectors $\mathbf{x}_{1}$, $\mathbf{x}_{2}, \cdots, \mathbf{x}_{N}$ in all possible combinations, repetitions allowed, for the vectors, $\mathbf{x}, \mathbf{y}, \cdots, \mathbf{t}$ in the invariants listed above.

The invariant of (2.7) involving nine vectors is also expressible as the sum of the 504 distinct expressions obtained from

$$
\sum x_{1} y_{1} z_{1} u_{1} v_{1}\left(w_{2} r_{2} s_{2} t_{3}-w_{3} r_{3} s_{3} t_{2}\right)
$$

by permuting the letters $x, \cdots, t$ in all possible ways. We note that $\theta(x, y, z, u ; v)$ is symmetric in the first four letters and that

$$
\begin{aligned}
\theta(x, y, z, u ; v)+\theta(v, x, y, z ; u)+\theta(u, v, x, y ; z) \\
+\theta(z, u, v, x ; y)+\theta(y, z, u, v ; x)=0 .
\end{aligned}
$$

Similarly, $\phi(x, y, z, u, v, w ; r)$ is symmetric in the first six letters and satisfies the equation

$$
\begin{gathered}
\phi(x, y, z, u, v, w ; r)+\phi(r, x, y, z, u, v ; w)+\phi(w, r, x, y, z, u ; v)+\phi(v, w, r, x, y, z ; u) \\
\quad+\phi(u, v, w, r, x, y ; z)+\phi(z, u, v, w, r, x ; y)+\phi(y, z, u, v, w, r ; x)=0 .
\end{gathered}
$$

3. Tensor basis. Let $L_{1}, \ldots, L_{R}$ denote the elements (2.7) of the integrity basis for $N$ vectors which are multilinear in the first $p, \cdots$, first $q$ vectors respectively. Then, the tensor basis is given [6] by the tensors

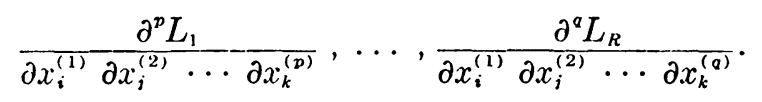

We shall employ the notation

$$
\begin{aligned}
c_{i j k l m}= & \sum \delta_{1 i} \delta_{1 j} \delta_{1 k}\left(\delta_{2 l} \delta_{3 m}-\delta_{3 l} \delta_{2 m}\right), \\
\theta_{i j k l m}= & c_{i j k l m}+c_{l i j k m}+c_{k l i j m}+c_{j k l i m}, \\
d_{i j k l m n p}= & \sum \delta_{1 i} \delta_{1 j} \delta_{1 k} \delta_{1 l} \delta_{1 m}\left(\delta_{2 n} \delta_{3 p}-\delta_{3 n} \delta_{2 p}\right), \\
\phi_{i j k l m n p}= & d_{i j k l m n p}+d_{n i j k l m p}+d_{m n i j k l p} \\
& +d_{l m n i j k p}+d_{k l m n i j p}+d_{i k l m n i p}
\end{aligned}
$$

where the term $\hat{o}_{1 i}$ is equal to one if $i=1$ and is equal to zero otherwise. Further, let $\tau_{i j k \text { mnpar }}$ denote the sum of the 504 distinct terms arising from

$$
\sum \delta_{1 i} \delta_{1 i} \delta_{1 k} \delta_{1 l} \delta_{1 m}\left(\delta_{2 n} \delta_{2 p} \delta_{2 q} \delta_{3 r}-\delta_{3 n} \delta_{3 p} \delta_{3 q} \delta_{2 r}\right)
$$

by permuting the subscripts $i, j, \cdots, r$ in all possible ways. We note that there are only 
five distinct tensors arising from $\theta_{i j k l m}$ upon permuting the subscripts due to the symmetry of $\theta_{i j k l m}$ in the first four subscripts. Only four of these five tensors are independent since

$$
\theta_{i j k l m}+\theta_{m i j k l}+\theta_{l m i j k}+\theta_{k l m i j}+\theta_{i k l m i}=0 .
$$

Similarly there are only seven distinct tensors arising from $\phi_{i j k l m n}$ upon permutation of the subscripts due to the symmetry of $\phi_{i j k l m n p}$ in the first six subscripts. Only six of these seven tensors are independent since

$$
\phi_{i j k l m n p}+\phi_{p i j k l m n}+\phi_{n p i j k l m}+\phi_{m n p i j k l}+\phi_{l m n p i j k}+\phi_{k l m n p i j}+\phi_{i k l m n p i}=0 \text {. }
$$

With (3.4) and (3.5) in mind, we see from (2.7), (3.1), (3.2) and (3.3) that the tensor basis for the gyroidal group is given by

$$
\begin{gathered}
\delta_{i j} ; e_{i j k} ; \sum \delta_{1 i} \delta_{1 j} \delta_{1 k} \delta_{1 l} ; \\
\theta_{i j k l m}, \theta_{m i j k l}, \theta_{l m i j k}, \theta_{k l m i j} ; \\
\sum \delta_{1 i} \delta_{1 j} \delta_{1 k} \delta_{1 l} \delta_{1 m} \delta_{1 n} ; \\
\phi_{i j k l m n p}, \phi_{p i j k l m n}, \phi_{n p i j k l m}, \phi_{m n p i j k l}, \phi_{l m n p i j k}, \phi_{k l m n p i j} ; \\
\tau_{i j k l m n p e r} .
\end{gathered}
$$

The tensors $\delta_{i j}$ and $e_{i j k}$ appearing in (3.6) are the Kronecker delta and the alternating tensor respectively. The tensors $\theta_{i j k l m}, \phi_{i j k l m n p}$ and $\tau_{i j k l m n p a r}$ are defined above.

Acknowledgment. This work was carried out under Grant Number NSF-GP-5709, through the National Science Foundation, at Brown University, Providence, Rhode Island.

\section{REFERENCES}

1. G. F. Smith and R. S. Rivlin, Integrity bases for vectors-the crystal classes, Arch. Rational Mech. Anal. $15,169(1964)$

2. (r. F. Smith, On the gencration of integrity bases, (to be published)

3. G. F. Smith and R. S. Rivlin, The strain-energy function for anisotropic elastic materials, Trans. Amer. Math. Soc. 88, $175(1958)$

4. H. Weyl, The classical groups, Princeton University Press, Princeton, N. J., 1946

5. H. W. 'Turnbull, The theory of determinants, matrices and invariants, Dover, New York, 1960

6. G. F. Smith and R. S. Rivlin, The anisotropic tensors, Quart. Appl. Math. 15, 308 (1957) 